\title{
Conducting Phase in the Two-Dimensional Disordered Hubbard Model
}

\author{
P. J. H. Denteneer \\ Lorentz Institute, University of Leiden, P.O. Box 9506, 2300 RA Leiden, The Netherlands \\ R. T. Scalettar \\ Physics Department, University of California, 1 Shields Avenue, Davis, California 95616 \\ N. Trivedi \\ Department of Theoretical Physics, Tata Institute of Fundamental Research, Homi Bhabha Road, Mumbai 400-005, India
}

(Received 1 April 1999)

\begin{abstract}
We study the temperature-dependent conductivity $\sigma(T)$ and spin susceptibility $\chi(T)$ of the twodimensional disordered Hubbard model. Calculations of the current-current correlation function using a quantum Monte Carlo method show that repulsion between electrons can significantly enhance the conductivity, and at low temperatures change the sign of $d \sigma / d T$ from positive (insulating behavior) to negative (conducting behavior). This result suggests the possibility of a metallic phase, and consequently a metal-insulator transition, in a two-dimensional microscopic model containing both interactions and disorder. The metallic phase is a non-Fermi liquid with local moments as deduced from $\chi(T)$.
\end{abstract}

PACS numbers: 71.10.Fd, 71.30.+h, 72.15.Rn

When electrons are confined to two spatial dimensions in a disordered environment, common understanding until recently was that the electronic states would always be localized and the system would therefore be an insulator. This idea is based on the scaling theory of localization [1] for noninteracting electrons and corroborated by subsequent studies using renormalization group (RG) methods [2]. The scaling theory highlights the importance of the number of spatial dimensions and demonstrates that while in three dimensions for noninteracting electrons there exists a transition from a metal to an Anderson insulator upon increasing the amount of disorder; a similar metal-insulator transition (MIT) is not possible in two dimensions.

The inclusion of interactions into the theory has been problematic, certainly when both disorder and interactions are strong and perturbative approaches break down. Following the scaling theory the effect of weak interactions in the presence of weak disorder was studied by diagrammatic techniques and found to increase the tendency to localize [3]. Subsequent perturbative RG calculations, including both electron-electron interactions and disorder, found indications of metallic behavior, but also, for the case without a magnetic field or magnetic impurities, found runaway flows to strong coupling outside the controlled perturbative regime and therefore were not conclusive [4,5]. The results of such approaches therefore have not changed the widely held opinion that in two dimensions (2D) the MIT does not occur.

The situation changed dramatically with the recent transport experiments on effectively 2D electron systems in silicon metal-oxide-semiconductor field-effect transistors (MOSFETs) which have provided surprising evidence that a MIT can indeed occur in 2D [6]. In these experiments the temperature dependence of the conductivity $\sigma_{\mathrm{dc}}$ changes from that typical of an insulator (decrease of $\sigma_{\mathrm{dc}}$ upon lowering $T$ ) at lower density to that typical of a conductor (increase of $\sigma_{\mathrm{dc}}$ upon lowering $T$ ) as the density is increased above a critical density. The fact that the data can be scaled onto two curves (one for the metal and one for the insulator) is seen as evidence for the occurrence of a quantum phase transition with carrier density $n$ as the tuning parameter. The possibility of such a transition has stimulated a large number of further experimental $[7,8]$ and also theoretical investigations $[9,10]$, including proposals that a superconducting state is involved [11]. Explanations in terms of trapping of electrons at impurities, i.e., not requiring a quantum phase transition, have also been put forward [12]. While there is no definitive explanation of the phenomena yet, it is likely that electron-electron interactions play an important role.

The central question motivated by the experiments is: Can electron-electron interactions enhance the conductivity of a 2D disordered electron system, and possibly lead to a conducting phase and a metal-insulator transition? It is this question that we address by studying the disordered Hubbard model which contains both relevant ingredients: interactions and disorder. While the Hubbard model does not include the long range nature of the Coulomb repulsion, studying the simpler model of screened interactions is an important first step in answering the central qualitative question posed above. We use quantum Monte Carlo simulation techniques which enable us to avoid the limitations of perturbative approaches (while of course being confronted with others). We mention that recent studies using very different techniques from ours have indicated that interactions may enhance conductivity: two interacting particles instead of one in a random potential have a delocalizing effect [13], and weak Coulomb interactions were found to increase the conductance of spinless electrons in (small) strongly disordered systems [14]. 
The disordered Hubbard model that we study is defined by

$$
\hat{H}=-\sum_{i, j, \sigma} t_{i j} c_{i \sigma}^{\dagger} c_{j \sigma}+U \sum_{j} n_{j \uparrow} n_{j \downarrow}-\mu \sum_{j, \sigma} n_{j \sigma},
$$

where $c_{j \sigma}$ is the annihilation operator for an electron at site $j$ with spin $\sigma . \quad t_{i j}$ is the nearest neighbor hopping integral, $U$ is the on-site repulsion between electrons of opposite spin, $\mu$ is the chemical potential, and $n_{j \sigma}=$ $c_{j \sigma}^{\dagger} c_{j \sigma}$ is the occupation number operator. Disorder is introduced by taking the hopping parameters $t_{i j}$ from a probability distribution $P\left(t_{i j}\right)=1 / \Delta$ for $t_{i j} \in[1-$ $\Delta / 2,1+\Delta / 2$ ], and zero otherwise. $\Delta$ is a measure for the strength of the disorder [15].

We use the determinant quantum Monte Carlo (QMC) method, which has been applied extensively to the Hubbard model without disorder [16]. While disorder and interaction can be varied in a controlled way and strong interaction is treatable, QMC is limited in the size of the lattice, and the sign problem restricts the temperatures which can be studied. The sign problem is minimized by choosing off-diagonal rather than diagonal disorder, as at least at half filling $(\langle n\rangle=1)$ there is no sign problem in the former case, and consequently simulations can be pushed to significantly lower temperatures. For results away from half filling we choose $\langle n\rangle=0.5$ where the sign problem is less severe compared to other densities [16]. Also, interestingly, the sign problem is reduced in the presence of disorder [15].

The quantity of immediate interest when studying a possible metal-insulator transition is the conductivity and especially its $T$ dependence. By the fluctuation-dissipation theorem $\sigma_{\mathrm{dc}}$ is related to the zero-frequency limit of the current-current correlation function. A complication of the QMC simulations is that the correlation functions are obtained as a function of imaginary time. To avoid a numerical analytic continuation procedure to obtain frequency-dependent quantities, which would require Monte Carlo data of higher accuracy than produced in the present study, we employ an approximation that was used and tested before in studies of the superconductorinsulator transition in the attractive Hubbard model [17]. This approximation is valid when the temperature is smaller than an appropriate energy scale in the problem. Additional checks and applicability to the present problem are discussed below. The approximation allows $\sigma_{\mathrm{dc}}$ to be computed directly from the wave vector $\mathbf{q}$ - and imaginary time $\tau$-dependent current-current correlation function $\Lambda_{x x}(\mathbf{q}, \tau)$ :

$$
\sigma_{\mathrm{dc}}=\frac{\beta^{2}}{\pi} \Lambda_{x x}(\mathbf{q}=0, \tau=\beta / 2) .
$$

Here $\beta=1 / T, \quad \Lambda_{x x}(\mathbf{q}, \tau)=\left\langle j_{x}(\mathbf{q}, \tau) j_{x}(-\mathbf{q}, 0)\right\rangle$, and $j_{x}(\mathbf{q}, \tau)$, the $\mathbf{q}, \tau$-dependent current in the $x$ direction, is the Fourier transform of $j_{x}(\ell)=i \sum_{\sigma} t_{\ell+\hat{x}, \ell} \times$ $\left(c_{\ell+\hat{x}, \sigma}^{\dagger} c_{\ell \sigma}-c_{\ell \sigma}^{\dagger} c_{\ell+\hat{x}, \sigma}\right)$ (see also Ref. [18]).
As a test for our conductivity formula (2) we first present results in Fig. 1(a) for $\sigma_{\mathrm{dc}}(T)$ at half filling for $U=4$ and various disorder strengths $\Delta$. The behavior of the conductivity shows that as the temperature is lowered below a characteristic gap energy the high $T$ "metallic" behavior crosses over to the expected low $T$ Mott insulating behavior for all $\Delta$, thereby providing a reassuring check of formula (2) and our numerics.

In Fig. 1(b), we show $\sigma_{\mathrm{dc}}(T)$ for a range of disorder strengths at density $\langle n\rangle=0.5$ and $U=4$. The figure displays a striking indication of a change from metallic behavior at low disorder to insulating behavior above a critical disorder strength, $\Delta_{\mathrm{c}} \simeq 2.7$. If this persists to
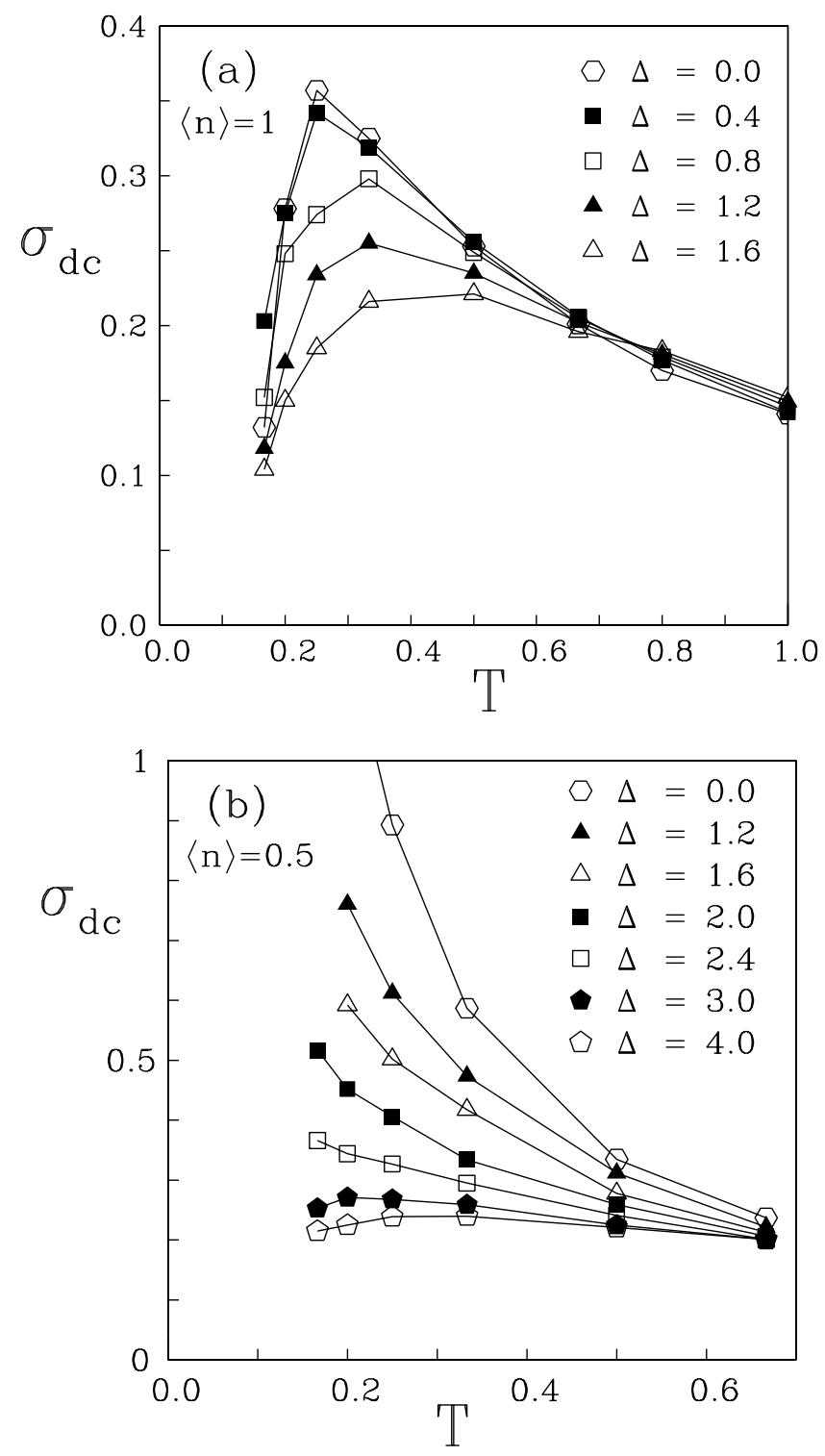

FIG. 1. Conductivity $\sigma_{\mathrm{dc}}$ as a function of temperature $T$ for various values of disorder strength $\Delta$ at $U=4$ for (a) half filling $(\langle n\rangle=1)$ and (b) $\langle n\rangle=0.5$. Calculations are performed on an $8 \times 8$ square lattice; data points are averages over four realizations for a given disorder strength. 
$T=0$ and in the thermodynamic limit, it would describe a ground state metal-insulator transition driven by disorder.

In order to obtain a more precise understanding of the role of interactions on the conductivity, we compare in Fig. 2 the results of Fig. 1(b) with the disordered noninteracting $\sigma_{0}$ [19]. The comparison is made at strong enough disorder $\Delta=2.0$ such that the localization length is less than the lattice size and the noninteracting system is therefore insulating with $d \sigma_{0} / d T>0$ at low $T$. Interactions are found to have a profound effect on the conductivity: in the high-temperature metallic region, interactions slightly reduce $\sigma$ compared to the noninteracting $\sigma_{0}$ behavior. On the other hand in the low-temperature "insulating" region of $\sigma_{0}$ the data show that upon turning on the Hubbard interaction the behavior is completely changed with $d \sigma / d T<0$, characteristic of metallic behavior. This is the regime of interest for the MIT.

In order to ascertain that the phase produced by repulsive interactions at low $T$ is not an insulating phase with a localization length larger than the system size but a true metallic phase we have studied the conductivity response for varying lattice sizes. We find a markedly different size dependence for the $U=0$ insulator and the $U=4$ metal, resulting in a confirmation of the picture given above. For $U=0$, the conductivity on a larger $(12 \times 12)$ system is lower than that on a smaller $(8 \times 8)$ system (see Fig. 2$)$, consistent with insulating behavior in the thermodynamic limit, whereas for $U=4$ the conductivity on the larger $(8 \times 8)$ system is higher than that on the smaller $(4 \times 4)$ system (data not shown), indicative of metallic behavior. Thus the enhancement of the conductivity by repulsive

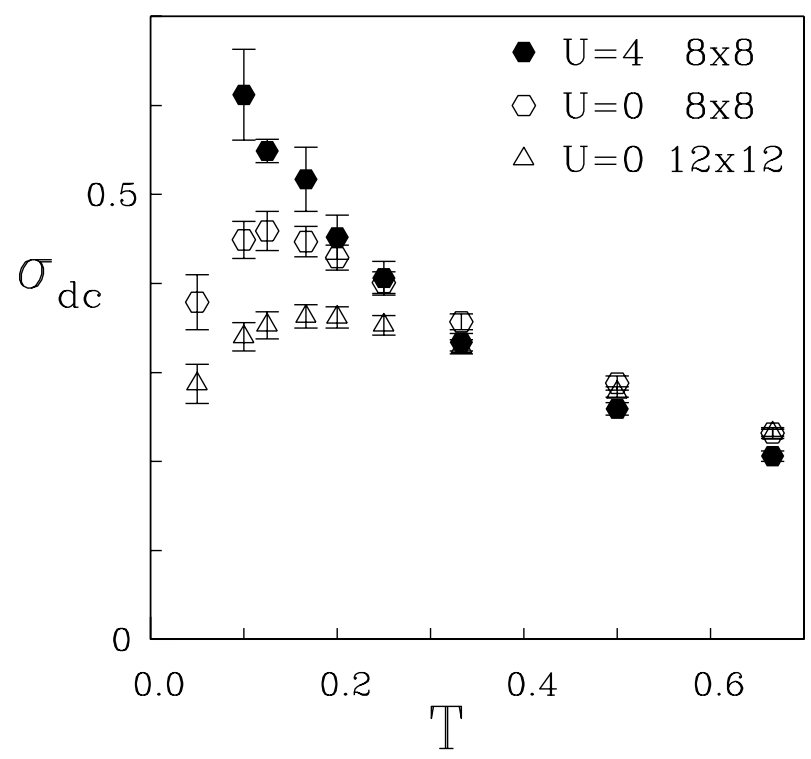

FIG. 2. Conductivity $\sigma_{\mathrm{dc}}$ as a function of temperature $T$ comparing $U=4$ and $U=0$ at $\langle n\rangle=0.5$ and disorder strength $\Delta=2.0$. Data points are averages over many realizations for this disorder strength (see text). Error bars are determined by the disorder averaging and not the Monte Carlo simulation. interactions becomes more pronounced with increased lattice size [20].

Concerning finite-size effects for the noninteracting system we note that at lower values of $\Delta$, where the localization length exceeds the lattice size, $\sigma_{0}$ shows metallic behavior which is diminished upon turning on the interactions [21]. Based on our analysis above, we would predict that at low enough $T$ and large enough lattice size the conductivity curves for the noninteracting $\sigma_{0}$ and interacting $\sigma$ cross with $\sigma>\sigma_{0}$ at sufficiently low $T$.

To obtain information on the spin dynamics of the electrons and because it is a quantity often discussed in connection with the localization transition, we also compute the spin susceptibility $\chi$ as a function of $T$ [through $\chi(T)=$ $\beta S_{0}(T)$ where $S_{0}$ is the magnetic structure factor at wave vector $\mathbf{q}=0$ ]. Figure 3 shows two things: (i) $\chi(T)$ is enhanced by interactions with respect to the noninteracting case (at fixed disorder strength), and (ii) starts to diverge when $T$ is lowered, both on the metallic $(\Delta=2)$ and insulating $(\Delta=4)$ sides of the alleged transition. This is in agreement with experimental and theoretical work on phosphorus-doped silicon, where a (3D) MIT is known to occur and the behavior is explained by the existence of local moments [22], and also with diagrammatic work on 2D disordered, interacting systems [23].

In order to definitively establish the existence of a possible quantum phase transition in the disordered Hubbard model requires the following: (i) extending the present data at $T=0.1=W / 80$, where $W$ is the noninteracting bandwidth, to lower $T$, which is however difficult because of the sign problem, (ii) a more detailed analysis of the

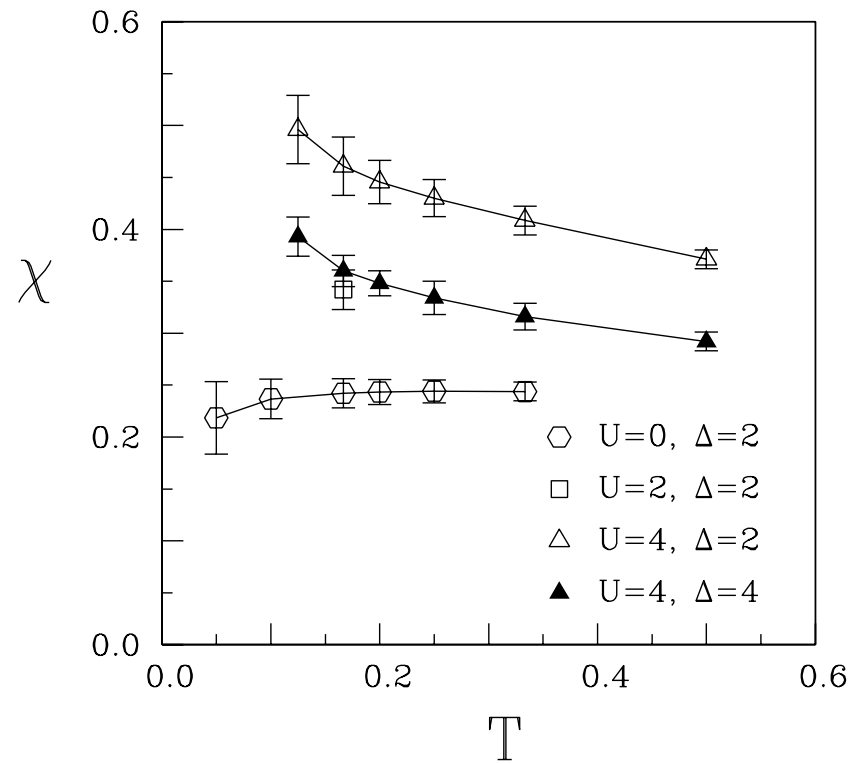

FIG. 3. Spin susceptibility $\chi$ as a function of temperature $T$ at $\langle n\rangle=0.5$ comparing interaction strengths $U=0,2,4$ and disorder strengths $\Delta=2,4$. Calculations are performed on $8 \times 8$ square lattices; error bars are from disorder averages over up to eight realizations. 
scaling behavior in both linear dimension and some scaled temperature, (iii) a more accurate analytic continuation procedure to extract the conductivity. The condition for the validity of the approximate formula (2) for $\sigma_{\mathrm{dc}}(T)$, requires that $T$ be less than an appropriate energy scale which is fulfilled within the two phases, but breaks down close to a quantum phase transition where the energy scale vanishes.

In summary, we have studied the temperature-dependent conductivity $\sigma(T)$ and spin susceptibility $\chi(T)$ of a model for two-dimensional electrons containing both disorder and interactions. We find that the Hubbard repulsion can enhance the conductivity and lead to a clear change in sign of $d \sigma / d T$. More significantly, from a finite-size scaling analysis we demonstrate that repulsive interactions can drive the system from one phase to a different phase. We find that $\sigma(T)$ has the opposite behavior as a function of system size in the two phases indicating that the transition is from a localized insulating to an extended metallic phase. The $\chi(T)$ data further suggest the formation of an unusual metal, a non-Fermi liquid with local moments. While the simplicity of the model we study prevents any quantitative connection to recent experiments on Si-MOSFETs, there is nevertheless an interesting qualitative similarity between Fig. 1(b) and the experiments. Varying the disorder strength $\Delta$ at fixed carrier density $\langle n\rangle$, as in our calculations, can be thought of as equivalent to varying carrier density at fixed disorder strength, as in experiments, since in a metal-insulator transition one expects no qualitative difference between tuning the mobility edge through the Fermi energy (by varying $\Delta$ ) and vice versa (by varying $\langle n\rangle$ ). Our work then suggests that electronelectron interaction induced conductivity plays a key role in the 2D metal-insulator transition.

We thank C. Huscroft for useful comments on the manuscript, H. V. Kruis for help with the calculations, and D. Belitz, R. N. Bhatt, C. Di Castro, T. R. Kirkpatrick, T. M. Klapwijk, S. V. Kravchenko, M.P. Sarachik, and G. T. Zimanyi for stimulating discussions. Work at UCD was supported by the SDSC, by the CLC program of UCOP, and by the LLNL Materials Institute.

[1] E. Abrahams, P. W. Anderson, D.C. Licciardello, and T. V. Ramakrishnan, Phys. Rev. Lett. 42, 673 (1979).

[2] F. Wegner, Z. Phys. B 36, 209 (1980); K. B. Efetov, A. I. Larkin, and D. E. Khmelnitskii, Sov. Phys. JETP 52, 568 (1980).

[3] P. A. Lee and T. V. Ramakrishnan, Rev. Mod. Phys. 57, 287 (1985).

[4] A. M. Finkel'stein, Zh. Eksp. Teor. Fiz. 84, 168 (1983) [Sov. Phys. JETP 57, 97 (1983)]; C. Castellani, C. Di Castro, P. A. Lee, and M. Ma, Phys. Rev. B 30, 527 (1984).

[5] D. Belitz and T. R. Kirkpatrick, Rev. Mod. Phys. 66, 261 (1994).
[6] S. V. Kravchenko, G. V. Kravchenko, J.E. Furneaux, V. M. Pudalov, and M. D'Iorio, Phys. Rev. B 50, 8039 (1994); S. V. Kravchenko, W.E. Mason, G. E. Bowker, J.E. Furneaux, V.M. Pudalov, and M. D'Iorio, Phys. Rev. B 51, 7038 (1995); S. V. Kravchenko, D. Simonian, M. P. Sarachik, W. Mason, and J. E. Furneaux, Phys. Rev. Lett. 77, 4938 (1996).

[7] D. Popović, A. B. Fowler, and S. Washburn, Phys. Rev. Lett. 79, 1543 (1997).

[8] D. Simonian, S. V. Kravchenko, and M. P. Sarachik, Phys. Rev. B 55, R13 421 (1997); Y. Hanien et al., Phys. Rev. Lett. 80, 1288 (1998); M. Y. Simmons et al., Phys. Rev. Lett. 80, 1292 (1998).

[9] V. Dobrosavljević, E. Abrahams, E. Miranda, and S. Chakravarty, Phys. Rev. Lett. 79, 455 (1997).

[10] C. Castellani, C. Di Castro, and P. A. Lee, Phys. Rev. B 57, R9381 (1998); S. Chakravarty, L. Yin, and E. Abrahams, Phys. Rev. B 58, R559 (1998); S. Chakravarty, S. Kivelson, C. Nayak, and K. Völker, e-print cond-mat/ 9805383.

[11] P. Phillips, Y. Wan, I. Martin, S. Knysh, and D. Dalidovich, Nature (London) 395, 253 (1998); D. Belitz and T. R. Kirkpatrick, Phys. Rev. B 58, 8214 (1998).

[12] B. L. Altshuler and D. Maslov, Phys. Rev. Lett. 82, 145 (1999); T. M. Klapwijk and S. Das Sarma, e-print condmat/9810349.

[13] D. L. Shepelyansky, Phys. Rev. Lett. 73, 2607 (1994); M. Ortuno and E. Cuevas, cond-mat/9808104.

[14] T. Vojta, F. Epperlein, and M. Schreiber, Phys. Rev. Lett. 81, 4212 (1998); X. Waintal, G. Benenti, and J.-L. Pichard, e-print cond-mat/9907318.

[15] M. Ulmke and R. T. Scalettar, Phys. Rev. B 55, 4149 (1997); M. Ulmke, P. J. H. Denteneer, R. T. Scalettar, and G. T. Zimanyi, Europhys. Lett. 42, 655 (1998).

[16] S. R. White, D. J. Scalapino, R. L. Sugar, E. Y. Loh, J. E. Gubernatis, and R.T. Scalettar, Phys. Rev. B 40, 506 (1989).

[17] N. Trivedi, R. T. Scalettar, and M. Randeria, Phys. Rev. B 54, R3756 (1996).

[18] D. J. Scalapino, S. R. White, and S. Zhang, Phys. Rev. B 47, 7995 (1993).

[19] We also consider lower temperatures and average over larger numbers of disorder realizations: for the higher temperatures generally 4 realizations are sufficient; for the lowest temperatures the fluctuations are larger and up to 20 (72) realizations are used for $U=4(U=0)$.

[20] For a related enhancement of the superfluid density by interactions in a disordered Bose system, see W. Krauth, N. Trivedi, and D. Ceperley, Phys. Rev. Lett. 67, 2307 (1991).

[21] For $\Delta=1.2$ and $\beta=5, \sigma_{\mathrm{dc}}=0.88(1), 0.76(1)$ for $U=0,4$, respectively. For $\Delta=2.0$ and $\beta=6, \sigma_{\mathrm{dc}}=$ $0.45(2), 0.49(3), 0.52(4)$ for $U=0,2,4$, respectively. Numbers between brackets represent the error bar in the last digit shown.

[22] M. A. Paalanen, S. Sachdev, R. N. Bhatt, and A.E. Ruckenstein, Phys. Rev. Lett. 57, 2061 (1986).

[23] C. Castellani, C. Di Castro, P. A. Lee, M. Ma, S. Sorella, and E. Tabet, Phys. Rev. B 30, 1596 (1984). 